\title{
A bacterial 'jack of all trades': Phototrophic Chloroflexi in hot spring environments and their impact on carbon cycling
}

\author{
AnNASTACIA C. BenNeTT ${ }^{1}$, TRINITY L. HAMILTON 1
}

1 University of Minnesota Plant and Microbial Biology Department, 1500 Gortner Avenue, St Paul, Minnesota

Phototrophic bacteria are key primary producers in terrestrial, alkaline hot springs, but factors that constrain the distribution of specific phototrophic taxa remain unresolved. Hot springs are extremely harsh environments, yet microbial communities of phototrophic Chloroflexi and Cyanobacteria have found ways to persist within these extremes. While Cyanobacteria ecology and physiology have been studied for years in Yellowstone National Park, representatives from phylum Chloroflexi remain understudied.

Cyanobacteria are experts in oxygenic photosynthesis and have been fine-tuning this process for millions of years, while the phototrophic Chloroflexi are physiologically and metabolically diverse. Chloroflexi have many tricks up their sleeves when it comes to carbon assimilation: they can be photoautotrophs, photoheterotrophs or photomixotrophs and rely on an array of different electron donors and acceptors commonly found in hot springs (H2S, $\mathrm{Fe}^{2+}$ and other inorganic compounds).

Here, we performed a cursory survey of Chloroflexi and Cyanobacteria by 16S rRNA gene sequencing biomass from seven sites along Rabbit Creek outflow in order to determine how specific taxa and diversity within these phyla changes along the natural temperature gradient. To assess photosynthetic activity, we set up carbon assimilation microcosm experiments with biomass from each site. Rabbit Creek, a circum-neutral geothermal stream in the Midway Geyser Basin of Yellowstone National Park, provides a natural temperature gradient ranging from a near boiling source to $\sim 45^{\circ} \mathrm{C}$ downstream. Additionally, Rabbit Creek has natural gradients of sulfide and $\mathrm{pH}$ and width of the stream expands downstream, leading to variable flow.

We found that while Cyanobacteria and a the photoheterotrophic Roseiflexus (Chloroflexi) remained relatively consistent between $55^{\circ} \mathrm{C}-65^{\circ} \mathrm{C}$, the rates of oxygenic photosynthesis decreased two-fold with increasing temperature. This suggests that either temperature or Roseiflexus could be impacting the physiology of Cyanobacteria. Previous work has shown that Cyanobacteria recovered from Yellowstone Hot Springs are active up to $72^{\circ} \mathrm{C}$, so it is likely that Chloroflexi could play a major role in the activity of Cyanobacteria, and the overall productivity of hot springs microbial communities in general. 